\title{
Method for Estimating Optimum Free Resonant Frequencies in Overcoupled WPT System
}

\author{
Dong-Wook Seo and Jae-Ho Lee \\ Automotive IT Platform Research Section, Electronics and Telecommunications Research Institute (ETRI), 1 Techno Sunhwan-ro 10-gil, \\ Yuga-myeon, Dalseong-gun, Daegu, Republic of Korea
}

Correspondence should be addressed to Jae-Ho Lee; jhlee1229@etri.re.kr

Received 30 January 2017; Revised 27 March 2017; Accepted 30 March 2017; Published 27 April 2017

Academic Editor: Marta Cavagnaro

Copyright (C) 2017 Dong-Wook Seo and Jae-Ho Lee. This is an open access article distributed under the Creative Commons Attribution License, which permits unrestricted use, distribution, and reproduction in any medium, provided the original work is properly cited.

\begin{abstract}
In our previous work, we proposed the method to maximize the output power even in the overcoupled state of the wireless power transfer (WPT) system by controlling free resonant frequencies and derived closed-form expression for optimum free resonant frequencies of the primary and secondary resonators. In this paper, we propose the mutual coupling approach to derive the optimum free resonant frequencies and show the measured power transfer efficiency (PTE) using the transmission efficiency as well as the system energy efficiency. The results of the proposed approach exactly coincide with those of the previous work, and the fabricated prototype achieves the transmission efficiency of about $80 \%$ by tuning the free resonant frequencies to the optimum values in the overcoupled state.
\end{abstract}

\section{Introduction}

Recently, the wireless power transfer (WPT) system has drawn a great deal of attention as it was applied in various electric devices including implanted devices [1], mobile devices [2], and electric vehicles [3]. Furthermore, many researchers are now starting to combine the WPT system with a communications system, and the combined system is called the simultaneous wireless information and power transfer (SWIPT) [4]. In the future, power lines are expected to be replaced by the WPT system. However, inductive WPT systems have the following inefficiency; it achieves the maximum output power and power transfer efficiency (PTE) only at a specific frequency and at a specific distance (i.e., coupling coefficient). Figure 1 shows the transmission coefficient of the WPT system of [5] as a function of frequency and as a function of coupling coefficient. For the operating frequency of $10 \mathrm{MHz}$, the frequency with the maximum transmission coefficient bifurcates at the critical coupling resulting in the wellknown frequency splitting phenomenon. The output power and the transmission coefficients are generally maximized at the critical coupling, and they decrease when the coupling coefficient deviates from the critical coupling, as shown in
Figure 1(b). In the overcoupled state, the excessive coupling between the coils results in frequency splitting, producing low transmission coefficients. On the other hand, low amount of power is transferred from the primary resonator to the secondary resonator in the undercoupled state, due to the low coupling coefficient. It is difficult to operate and maintain the WPT system at the critical coupling. According to changed operating circumstances, that is to say, changed coupling coefficient, some system parameters should be adjusted to achieve the high transmission coefficient.

Figure 2 shows an equivalent circuit model of a twocoil WPT system, where the controllable elements include voltage source $V_{s}$, source impedance $R_{s}$, load impedance $R_{L}$, primary coil $L_{1}$, secondary coil $L_{2}$, and capacitances of primary and secondary resonators, $C_{1}$ and $C_{2}$. Depending on the circuit elements, various methods have been introduced to improve the PTE and output power of the WPT system [613]. These methods are categorized in two types: a method that changes the critical coupling coefficient [6-10] and a method that mitigates the equivalent coupling coefficient in the overcoupled state [11-13]. The former methods control the resistance or inductance in order to change the critical coupling coefficient $k_{c}$, since $k_{c}$ is a function of the resistance 


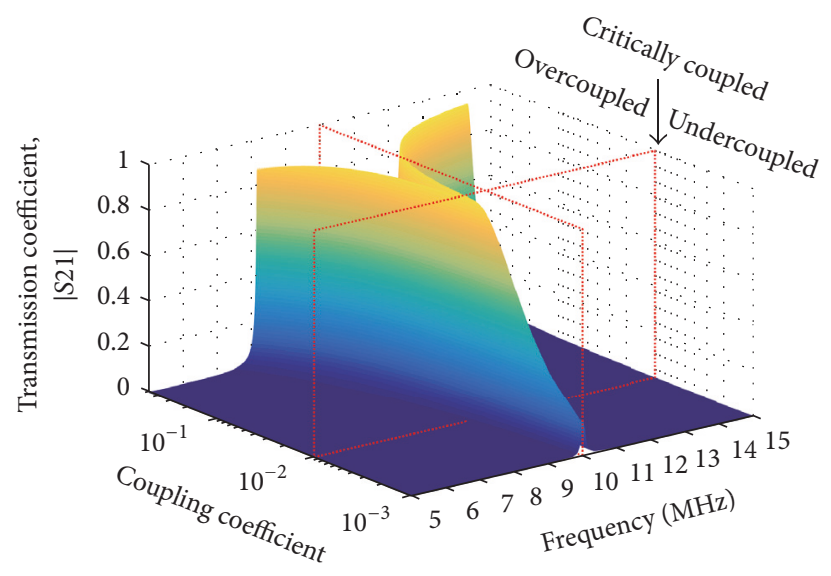

(a)

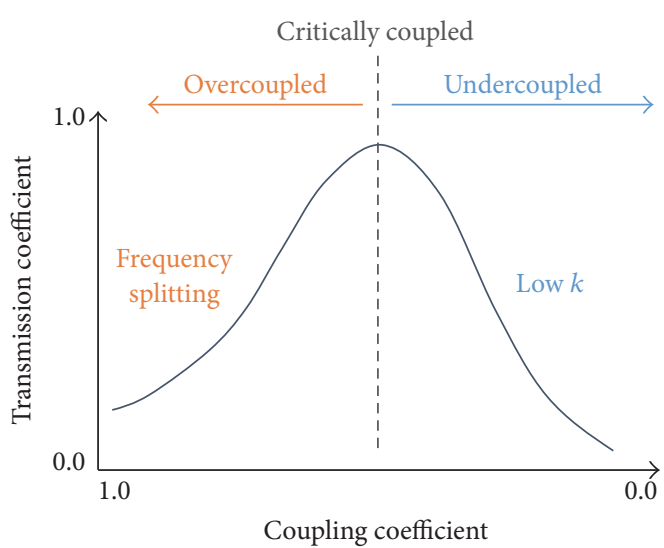

(b)

FIGURE 1: Transmission coefficient of a WPT system (a) as a function of frequency and coupling coefficient and (b) as a function of coupling coefficient at the operating frequency of $10 \mathrm{MHz}$.

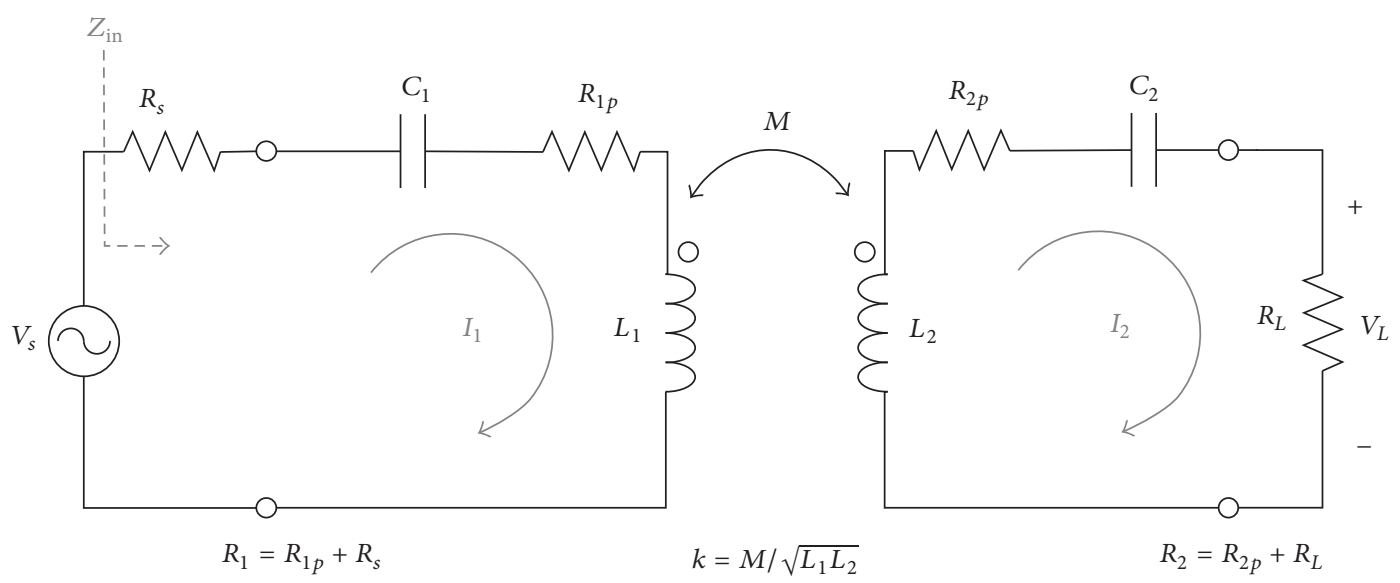

FIgURE 2: Equivalent circuit model of a two-coil resonant WPT system.

and inductance. The latter methods control the voltage source or capacitance to change the operating frequency or free resonant frequencies only in the overcoupled state.

For the control of resistance, the most commonly used method is the matching networks. This method converts source and load impedances into optimum values. Our group revealed that the WPT system using lumped reactive matching networks is equivalent to a two-coil WPT system, and the WPT system using transformer matching networks could be considered as a four-coil WPT system [6]. Additionally, we showed how the source (or load) impedance could be matched to the optimum value not only in the two-coil WPT system but also in the four-coil WPT system. In [7], a switchable capacitor array circuit was proposed, and it was shown that several capacitors were enough to implement a variety of matching cases in an $L$-section matching network. Our group showed that the WPT system can achieve the high PTE by adjusting only the source impedance [8], and we also demonstrated the self-adaptive WPT system achieving the high PTE without the feedback of receiver information [9].
Besides the method using matching networks, the method of selecting the optimum primary and secondary coils among the coil arrays was suggested in [10].

By adjusting either the amplitude or the operating frequency of voltage source, the output power can be improved. Changing the amplitude of the voltage source does not improve the PTE, while changing the operating frequency in overcoupled state achieves the high PTE [11, 12]. However, this method may result in the violation of frequency regulation for a WPT system in narrow bandwidth. Similarly, our group recently proposed that free resonant frequencies of resonators are tuned to optimum frequencies in order to achieve a high PTE in overcoupled state [13]. In [13], the optimum free resonant frequencies were derived in the closed-form based on a condition having a current ratio between the primary and secondary resonators identical to that in critical coupled state. In addition, the system energy efficiency was used to express the PTE instead of the more commonly used transmission efficiency. On the other hand, the free resonant frequency tuning method was 
applied not only to the two-coil WPT system but also to the multicoil WPT system [14]. The authors of [14] determined the capacitance and free resonant frequencies by using a genetic algorithm; however, the process does not guarantee a global maximum and takes dramatically increasing time as the number of resonators increases. Additionally, it is also cumbersome to apply the principles used in [13] to the multicoil WPT system.

In [15], we have already presented the idea that the effect of mutual inductance on a reflected inductance can be used to derive the condition of the optimum free resonant frequencies for a high PTE. In this paper, we form the concept in greater detail and show that the tuned free resonant frequencies result in a high PTE using the transmission efficiency as well as the output power.

\section{Formulation}

In the circuit model of Figure 2, $M$ is the mutual inductance between the primary and secondary coils and is given by

$$
M=k \sqrt{L_{1} L_{2}}, \quad 0 \leq k \leq 1,
$$

where $k$ is the coupling coefficient.

When no mutual coupling exists, that is, $M=k=0$, the resonance frequency is called the free resonant frequency. The free resonance frequencies of the primary and secondary resonators are given, respectively,

$$
\begin{aligned}
& \omega_{1}=\frac{1}{\sqrt{L_{1} C_{1}}}, \\
& \omega_{2}=\frac{1}{\sqrt{L_{2} C_{2}}} .
\end{aligned}
$$

In the conventional WPT system, the free resonant frequencies are tuned to the operating frequency, $\omega_{0}$, allowing the WPT system to function at the operating frequency. However, as the definition of the free resonant frequency might suggest, the primary and secondary resonators work at the operating frequency only when there is little or no mutual coupling. If the mutual coupling becomes stronger, then the resonators affect each other, and the resonant frequencies of the resonators are no longer equal to the operating frequency. To see this effect intuitively, we use reflected impedance concept. The circuit model of Figure 2 can be described using the reflected impedance $Z_{r}$ as shown in Figure 3, and $Z_{r}$ is expressed as

$$
Z_{r}=\frac{\omega^{2} M^{2}}{R_{L}+R_{2 p}+j \omega L_{2}+1 / j \omega C_{2}} .
$$

The loaded $Q$ of the primary and secondary series RLC resonators are defined as, respectively,

$$
\begin{aligned}
& Q_{1}=\frac{\omega L_{1}}{R_{s}+R_{1 p}}, \\
& Q_{2}=\frac{\omega L_{2}}{R_{L}+R_{2 p}} .
\end{aligned}
$$

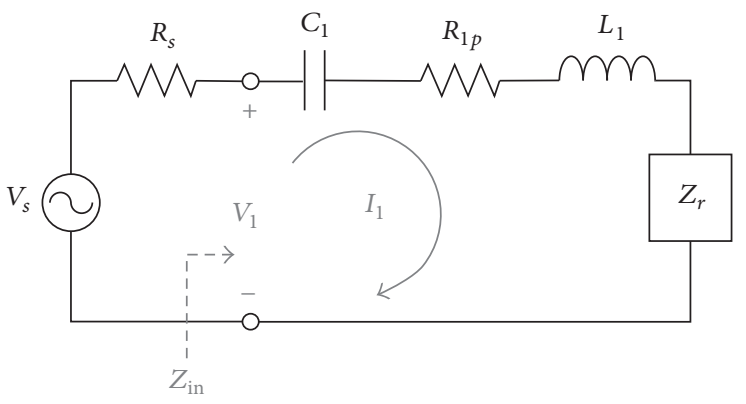

FIGURE 3: Equivalent circuit model of a two-coil resonant WPT system using reflected impedance.

Using (1), (2), and (4), $Z_{r}$ can be rewritten as

$$
Z_{r}=\frac{\omega L_{1} k^{2}}{1 / Q_{2}+j\left(1-\omega_{2}^{2} / \omega^{2}\right)},
$$

where the real and imaginary parts are given by

$$
\begin{aligned}
& \operatorname{Re}\left\{Z_{r}\right\}=\omega L_{1} \cdot \frac{k^{2} / Q_{2}}{1 / Q_{2}^{2}+\left(1-\omega_{2}^{2} / \omega^{2}\right)^{2}}, \\
& \operatorname{Im}\left\{Z_{r}\right\}=\omega L_{1} \cdot \frac{k^{2}\left(\omega_{2}^{2} / \omega^{2}-1\right)}{1 / Q_{2}^{2}+\left(\omega_{2}^{2} / \omega^{2}-1\right)^{2}} .
\end{aligned}
$$

Therefore, due to the mutual coupling, the resistance of (6) and reactance of (7) are added to the primary resonator. Let the right-hand side of (7) be the product of $\omega L_{1}$ and a function of the free resonant frequency of the secondary resonator, $A\left(\omega_{2}\right)$. Then, the total inductance of the primary resonator becomes $L_{1}+L_{1} \cdot A\left(\omega_{2}\right)$. In order to work at the operating frequency, the resonant frequency of the primary resonator should be

$$
\frac{1}{\sqrt{L_{1}\left[1+A\left(\omega_{2}\right)\right] \cdot C_{1}}}=\frac{\omega_{1}}{\sqrt{1+A\left(\omega_{2}\right)}}=\omega_{0},
$$

which is satisfied by adjusting the capacitance of the primary resonator, $C_{1}$.

Similarly, in order to operate the secondary resonator at the operating frequency $\omega_{0}$, the resonant frequency of the secondary resonator should also be

$$
\frac{1}{\sqrt{L_{2}\left[1+B\left(\omega_{1}\right)\right] \cdot C_{2}}}=\frac{\omega_{2}}{\sqrt{1+B\left(\omega_{1}\right)}}=\omega_{0},
$$

where $B\left(\omega_{1}\right)$ is given by

$$
B\left(\omega_{1}\right)=\frac{k^{2}\left(\omega_{1}^{2} / \omega^{2}-1\right)}{1 / Q_{1}^{2}+\left(\omega_{1}^{2} / \omega^{2}-1\right)^{2}} .
$$

Since variables $A\left(\omega_{2}\right)$ and $B\left(\omega_{1}\right)$ are functions of $\omega_{2}$ and $\omega_{1}$, respectively, (8) and (9) must be solved simultaneously for the variables $\omega_{2}$ and $\omega_{1}$. 


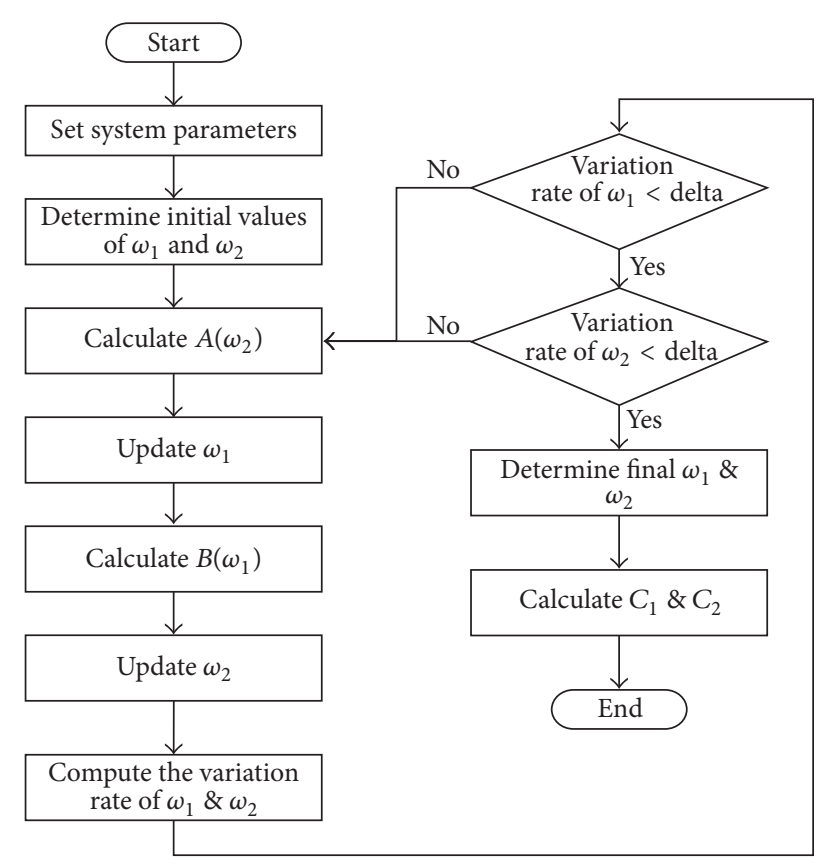

FIGURE 4: Flowchart of the proposed iterative process.

\section{Estimation Procedure}

It is cumbersome to directly solve the simultaneous equations (8) and (9). Instead, we can find the optimal free resonant frequencies using an iterative process, as summarized in Figure 4. The detailed steps of the proposed scheme are described as follows.

(1) Set the operating frequency $\omega_{0}$ and system parameters $R_{s}, R_{1 p}, L_{1}, R_{L}, R_{2 p}, L_{2}$, and $k$ of Figure $2 . V_{s}, C_{1}$, and $\mathrm{C}_{2}$ are not required.

(2) It is recommended that the initial $\omega_{1}$ and $\omega_{2}$ are set to be two times the operating frequency. If the initial $\omega_{1}$ and $\omega_{2}$ are set to be $\omega_{0}, A\left(\omega_{2}\right)$ and $B\left(\omega_{1}\right)$ terms become zero, which removes the mutual coupling in the reactance of the reflected impedance.

(3) Calculate $A\left(\omega_{2}\right)$ using (7) and update $\omega_{1}$ using (8).

(4) Calculate $B\left(\omega_{1}\right)$ using (10) and update $\omega_{2}$ using (9).

(5) Compute the variation of $\omega_{1}$ and $\omega_{2}$. If $\Delta \omega_{1} / \omega_{1}$ and $\Delta \omega_{2} / \omega_{2} \leq 0.1 \%$, then go to step (6). Otherwise, go to step (3).

(6) Obtain the final $\omega_{1}$ and $\omega_{2}$.

(7) Estimate $C_{1}$ and $C_{2}$ by using (2) according to the final $\omega_{1}$ and $\omega_{2}$.

\section{Simulation and Experiment Results}

We simulated and measured the WPT system with the identical parameter used in [13]. Figure 5 shows schematics and component values of the experimental setup to validate the proposed method. The capacitance values of the primary

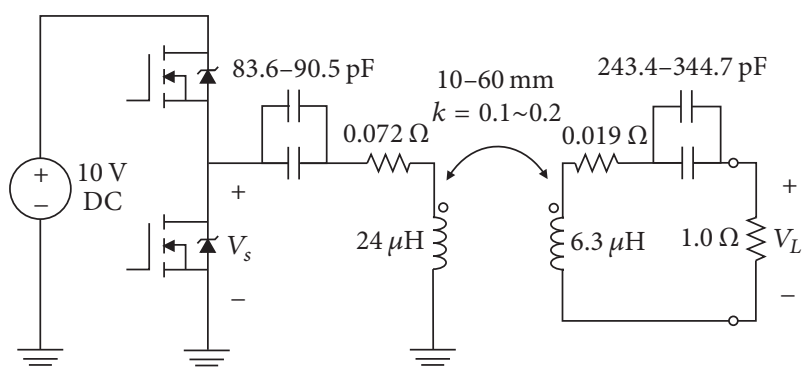

FIGURE 5: Schematic of the WPT system prototype.

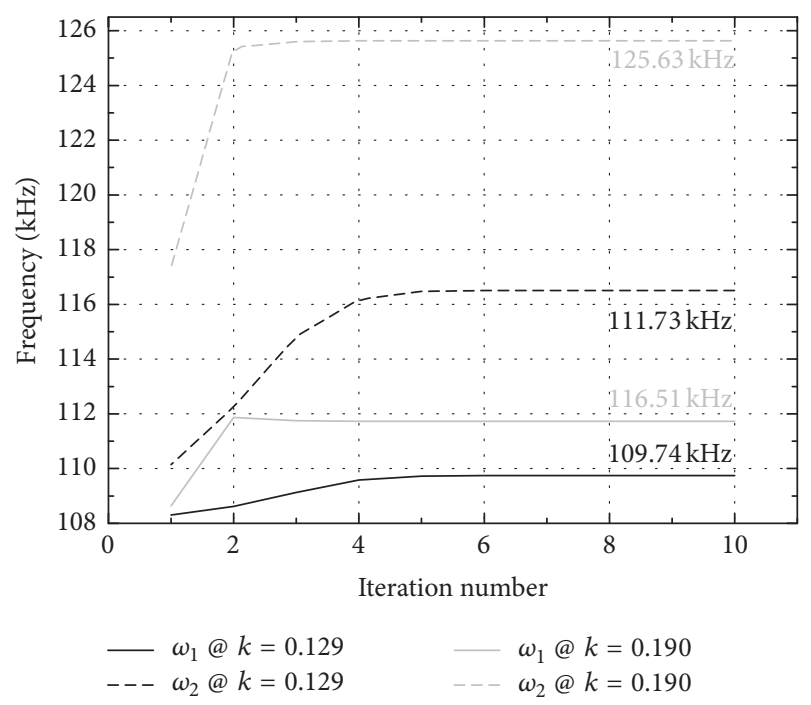

FIGURE 6: Free resonant frequencies with respect to iteration number for $k=0.129$ and 0.190 .

and secondary resonators are varied to adjust the free resonant frequencies to the estimated optimum values in the overcoupled state. The switching frequency of the seriesresonant half-bridge inverter is $108 \mathrm{kHz}$, and IR25603 and IRFP250N are used as the inverter driver and the switching device, respectively. The coils that were used were obtained from ABRACON Corp. Because of high voltage, the film capacitors and cement resistors were used as the capacitor of the resonators, the source, and load resistors.

Figure 6 shows the estimated free resonant frequencies with respect to the iteration number. We show two cases of the overcoupled state, $k=0.129$ and 0.190 . In this system, $Q_{1}$ and $Q_{2}$ are 21.1 and 4.2, respectively, since $Q_{1} \neq Q_{2}, \omega_{1} \neq \omega_{2}$. Additionally, the higher the loaded $Q$ of the other resonator is, the more the free resonant frequency deviates from $\omega_{0}$. The optimum free resonant frequencies are reached only after several iterations.

We summarized the results of [13] and the proposed iterative process in Table 1. The estimated optimum free resonant frequencies using the proposed method are very similar to those of [13]. We highlight the fact that there is only a maximum difference of $0.04 \mathrm{kHz}$ between the results of two methods. 


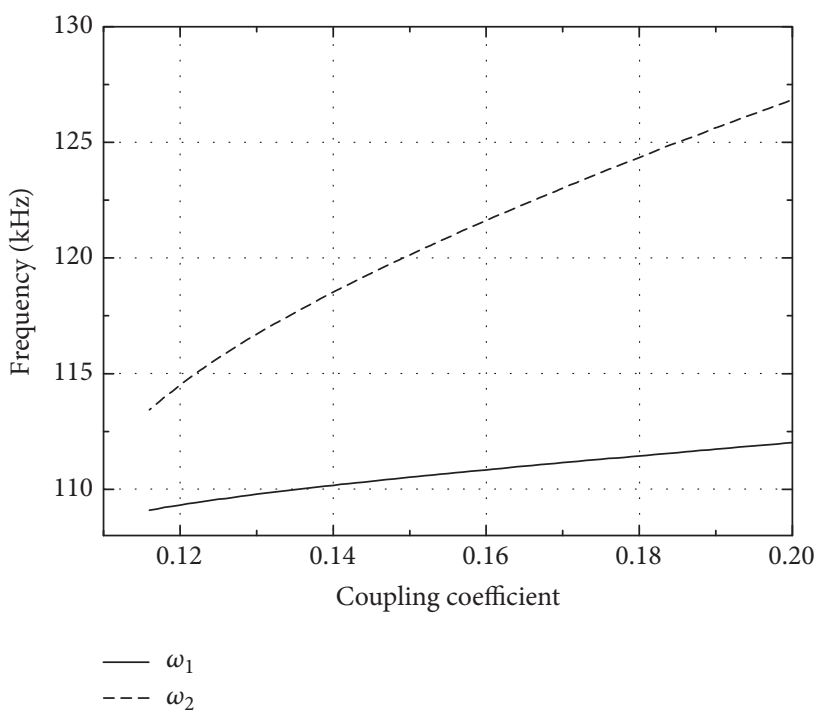

(a)

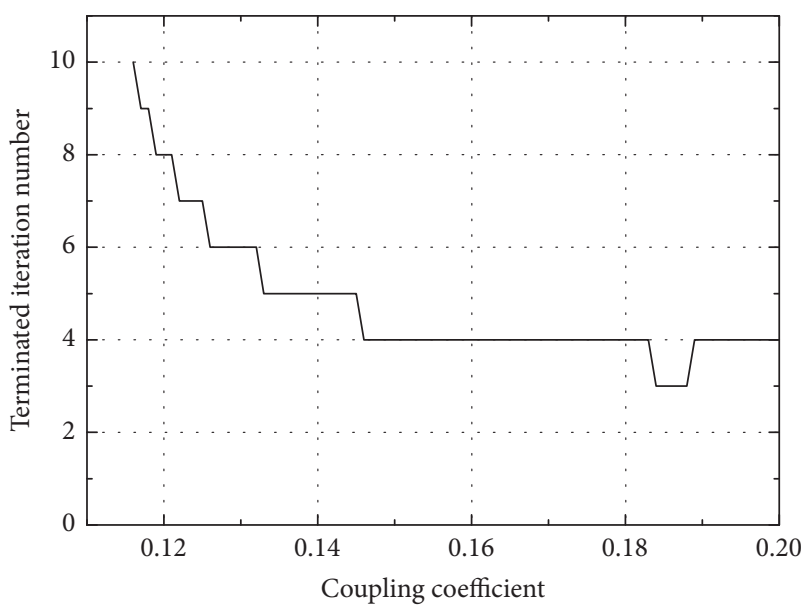

(b)

FIGURE 7: Estimated results using the proposed method as a function of coupling coefficient: (a) free resonant frequencies and (b) terminated iteration number.

TABLE 1: The estimated free resonant frequencies using method of [13] and the proposed method with respect to coupling coefficients to achieve the high PTE.

\begin{tabular}{|c|c|c|c|c|}
\hline $\begin{array}{l}\text { Coupling } \\
\text { coefficient }\end{array}$ & $\begin{array}{c}\omega_{1} \& \omega_{2} \text { of } \\
{[13][\mathrm{kHz}]}\end{array}$ & $\begin{array}{c}\omega_{1} \& \omega_{2} \\
\text { using the } \\
\text { proposed } \\
\text { method } \\
{[\mathrm{kHz}]}\end{array}$ & $\begin{array}{c}\text { Difference } \\
{[\mathrm{kHz}]}\end{array}$ & $\begin{array}{c}\text { Terminated } \\
\text { iteration } \\
\text { number }\end{array}$ \\
\hline \multirow{2}{*}{0.214} & 112.38 & 112.39 & 0.01 & \multirow{2}{*}{5} \\
\hline & 128.54 & 128.51 & 0.03 & \\
\hline \multirow{2}{*}{0.190} & 111.73 & 111.73 & 0 & \multirow{2}{*}{4} \\
\hline & 125.63 & 125.63 & 0 & \\
\hline \multirow{2}{*}{0.164} & 110.97 & 110.97 & 0 & \multirow{2}{*}{4} \\
\hline & 122.19 & 122.19 & 0 & \\
\hline \multirow{2}{*}{0.143} & 110.28 & 110.28 & 0 & \multirow{2}{*}{5} \\
\hline & 119.02 & 119.02 & 0 & \\
\hline \multirow{2}{*}{0.129} & 109.75 & 109.74 & 0.01 & \multirow{2}{*}{6} \\
\hline & 116.51 & 116.51 & 0 & \\
\hline \multirow{2}{*}{0.116} & 109.11 & 109.10 & 0.01 & \multirow{2}{*}{10} \\
\hline & 113.48 & 113.44 & 0.04 & \\
\hline
\end{tabular}

Figure 7 shows the estimated optimum free resonant frequencies of the primary and secondary resonators as a function of the coupling coefficient, and the iteration number when the proposed process is terminated. As expected, the larger the coupling coefficient is, the farther away the optimum free resonant frequencies deviate from the operating frequency $(108 \mathrm{kHz})$. The interesting point is that the smaller the coupling coefficient is, the more the iterations were needed. We hypothesize that it is because the initial values of $\omega_{1}$ and $\omega_{2}$ are set to be two times $\omega_{0}$. Nevertheless, convergence is reached within ten iterations.
The efficiency (or power gain) of an electrical system is defined as the ratio of an output power to an input power. The output power is referred to as a load power, while the input power is defined as either the total input power from the power source, $V_{s} \cdot I_{1}{ }^{*}$, or maximum available power at the power source, $\left|V_{s}\right|^{2} / 4 R_{s}$. Efficiency is defined differently depending on the definition of the input power. According to [16], the efficiency with the former notation is called "system energy efficiency" which is given by $\eta=\left(\left|V_{L}\right|^{2} / R_{L}\right) /\left(V_{s} \cdot I_{1}{ }^{*}\right)$, and the efficiency with the latter notation is called "transmission efficiency" which is given by $\eta=\left(\left|V_{L}\right|^{2} / R_{L}\right) /\left(\left|V_{s}\right|^{2} / 4 R_{s}\right)$. The difference between them is determined by whether the power loss by the source resistance is included in the input power or not. In general, the system energy efficiency is used in the WPT with negligible source resistance and low operation frequency, while the transmission efficiency is used in the WPT operating at a high frequency and with fixed source resistance. In this study, we use the transmission efficiency to represent the performance of the WPT system, because $V_{s}$ and $R_{s}$ of the generator were fixed as $4.5 \mathrm{Vrms}$ and $0.7 \Omega$, respectively.

Figure 8 depicts the measured load power and transmission efficiency when the values are optimized for the tuned free resonant frequencies obtained from the proposed process. Since the input power in the transmission efficiency is defined as the maximally delivered power from the power source, the input power keeps unchanged regardless of status of the WPT system and load. Then, the transmission efficiency has a direct relation to the load power. From Figure 8, we notice that the transmission efficiency is maximized by maximizing the load power. When the tuned free resonant frequencies are applied to the overcoupled WPT system, the system energy efficiency approaches about $50 \%$ in [13] while the transmission efficiency approaches about $81 \%$. Obviously, the tuned free resonant frequencies effectively improve the 


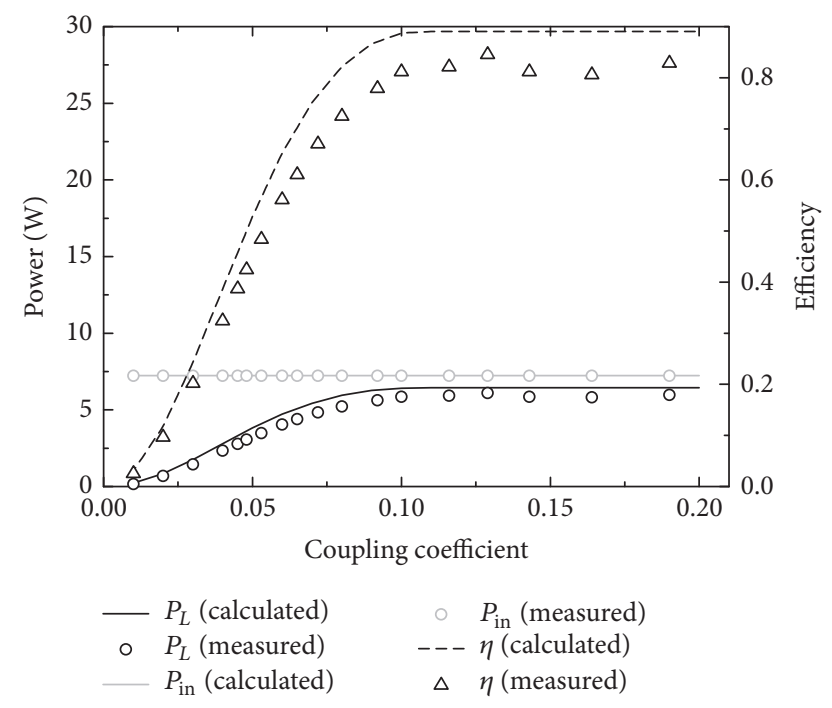

FIGURE 8: Calculated and measured load power and transmission efficiency.

transmission efficiency and load power in the overcoupled state.

\section{Conclusion}

In this paper, we proposed a new approach to derive the optimum free resonant frequencies in the overcoupled WPT system. The optimum values can be obtained through several iterations and are identical to those obtained in previous works. Additionally, when the tuned free resonant frequencies are adopted in the overcoupled WPT system, the system achieves the transmission efficiency close to $80 \%$ and the system energy efficiency close to $50 \%$.

The proposed method is very intuitive and simple. Therefore, it can be extended to the sophisticated applications such as a multicoil WPT system.

\section{Conflicts of Interest}

The authors declare that there are no conflicts of interest regarding the publication of this paper.

\section{Acknowledgments}

This work was supported by ETRI R\&D Program [17ZD1200, Advanced Development of Fusion-Platform and Local Parts Maker Support Project for Context-Aware Smart Vehicle] funded by the Government of Korea.

\section{References}

[1] D.-W. Seo, J.-H. Lee, and H. Lee, "Integration of resonant coil for wireless power transfer and implantable antenna for signal transfer," International Journal of Antennas and Propagation, vol. 2016, Article ID 7101207, 7 pages, 2016.
[2] E. Waffenschmidt, "Wireless power for mobile devices," in Proceedings of the IEEE 33rd International Telecommunications Energy Conference (INTELEC '11), pp. 1-9, Amsterdam, The Netherlands, October 2011.

[3] S. Moon and G.-W. Moon, "Wireless power transfer system with an asymmetric four-coil resonator for electric vehicle battery chargers," IEEE Transactions on Power Electronics, vol. 31, no. 10, pp. 6844-6854, 2016.

[4] Z. H. Liu, W. J. Xu, S. Y. Li, C. Z. Long, and J. R. Lin, "Simultaneous information and power transfer for multi-antenna primary-secondary cooperation in cognitive radio networks," ETRI Journal, vol. 38, no. 5, pp. 941-951, 2016.

[5] A. P. Sample, D. A. Meyer, and J. R. Smith, "Analysis, experimental results, and range adaptation of magnetically coupled resonators for wireless power transfer," IEEE Transactions on Industrial Electronics, vol. 58, no. 2, pp. 544-554, 2011.

[6] D.-W. Seo, J.-H. Lee, and H. Lee, "Study on two-coil and four-coil wireless power transfer system using Z-parameter approach," ETRI Journal, vol. 38, no. 3, pp. 568-578, 2016.

[7] Y. Lim, H. Tang, S. Lim, and J. Park, "An adaptive impedancematching network based on a novel capacitor matrix for wireless power transfer," IEEE Transactions on Power Electronics, vol. 29, no. 8, pp. 4403-4413, 2014.

[8] D.-W. Seo, J.-H. Lee, and H. Lee, "Method for adjusting single matching network for high power transfer efficiency of wireless power transfer system," ETRI Journal, vol. 38, no. 5, pp. 962-971, 2016.

[9] D.-W. Seo, S.-T. Khang, S.-C. Chae, J.-W. Yu, G.-K. Lee, and W.S. Lee, "Open-loop self-adaptive wireless power transfer system for medical implants," Microwave and Optical Technology Letters, vol. 58, no. 6, pp. 1271-1275, 2016.

[10] J. Kim and J. Jeong, "Range-adaptive wireless power transfer using multiloop and tunable matching techniques," IEEE Transactions on Industrial Electronics, vol. 62, no. 10, pp. 6233-6241, 2015.

[11] N. Y. Kim, K. Y. Kim, J. Choi, and C.-W. Kim, "Adaptive frequency with power-level tracking system for efficient magnetic resonance wireless power transfer," Electronics Letters, vol. 48, no. 8, pp. 452-454, 2012.

[12] Y. Luo, Y. Yang, S. Chen, and X. Wen, "A frequency-tracking and impedance-matching combined system for robust wireless power transfer," International Journal of Antennas and Propagation, vol. 2017, Article ID 5719835, 13 pages, 2017.

[13] D.-W. Seo, J.-H. Lee, and H.-S. Lee, "Optimal coupling to achieve maximum output power in a WPT system," IEEE Transactions on Power Electronics, vol. 31, no. 6, pp. 3994-3998, 2016.

[14] B.-H. Choi and J.-H. Lee, "Design of asymmetrical relay resonators for maximum efficiency of wireless power transfer," International Journal of Antennas and Propagation, vol. 2016, Article ID 8247476, 8 pages, 2016.

[15] D.-W. Seo, J.-H. Lee, and M.-R. Park, "High-efficiency wireless power transfer by controlling free resonant frequencies," in Proceedings of the International Symposium on Antennas and Propagation (ISAP '16), pp. 518-519, Ginowan, Japan, October 2016.

[16] S. Y. R. Hui, W. Zhong, and C. K. Lee, "A critical review of recent progress in mid-range wireless power transfer," IEEE Transactions on Power Electronics, vol. 29, no. 9, pp. 4512-4520, 2014. 


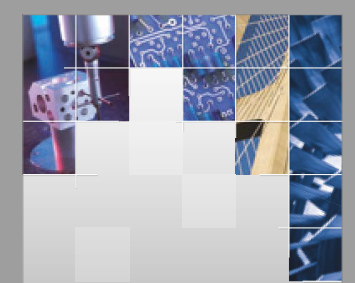

\section{Enfincering}
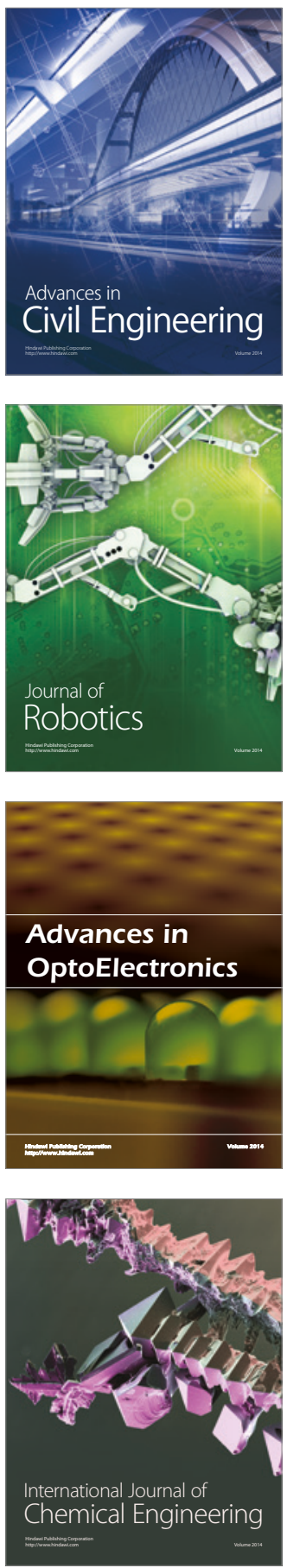

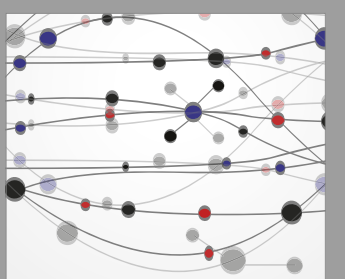

The Scientific World Journal

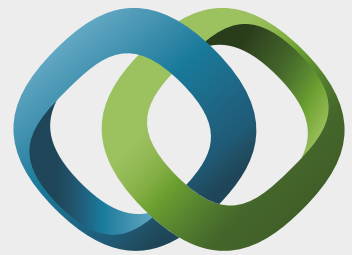

\section{Hindawi}

Submit your manuscripts at

https://www.hindawi.com
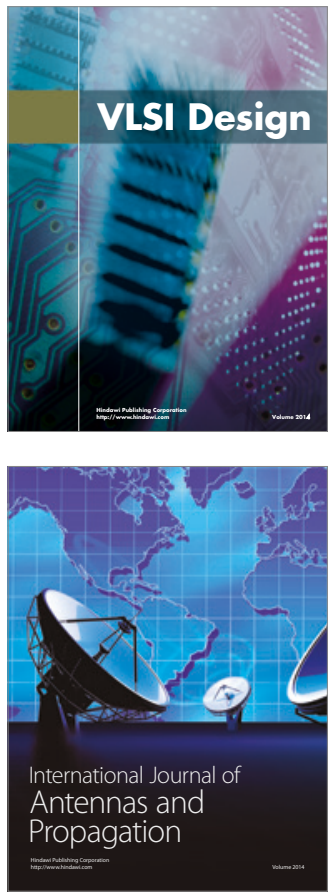

\section{Rotating}

Machinery
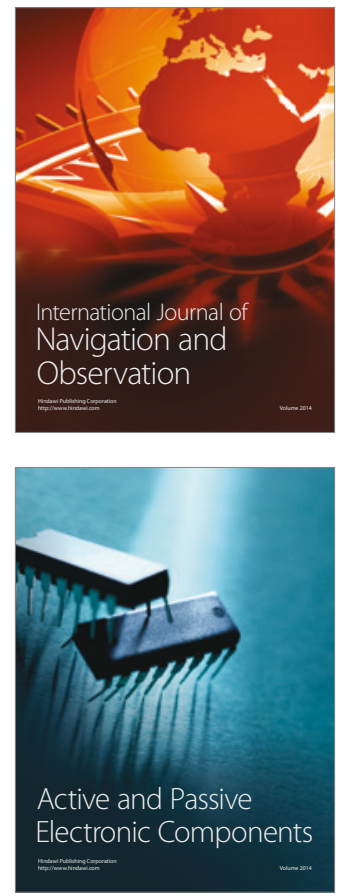
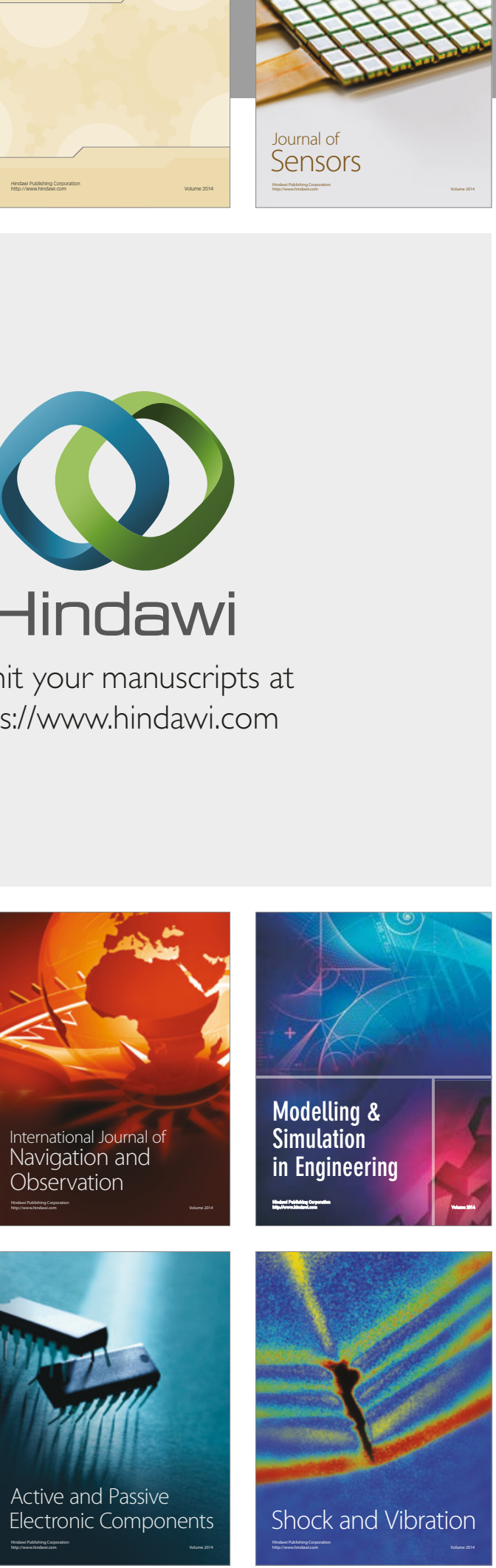
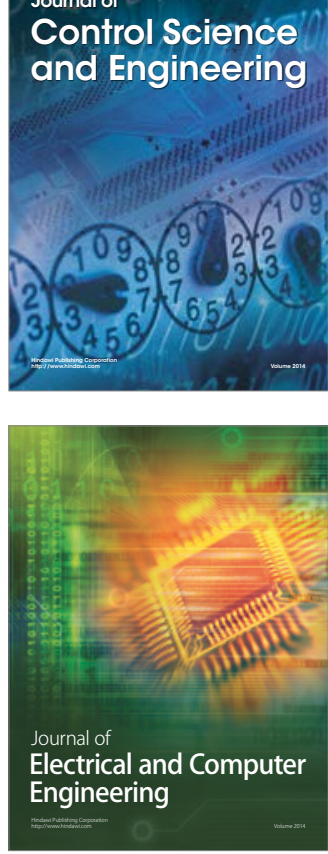

Distributed

Journal of

Control Science

and Engineering
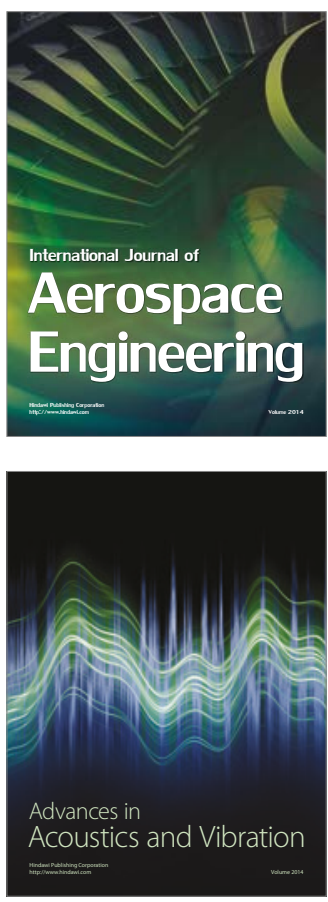

Sensor Networks 